\title{
Modified Lateral Crural Tensioning: Improving the Tip Without Articulated Alar Rim Grafts
}

\author{
Guilherme Constante Preis Sella ${ }^{1, ~ *, ~ B a ́ r b a r a ~ d a s ~ N e v e s ~ L i n h a r e s ², ~}$ \\ Mario Bazanelli Junqueira Ferraz ${ }^{2}$ \\ ${ }^{1}$ Clínica Avançada de Otorrinolaringologia, Facial Plastic Surgery, Maringá, Brasil \\ ${ }^{2}$ Clinica Mario Ferraz, Facial Plastic Surgery, Campinas, Brasil

\section{Email address:} \\ guilherme_sella@yahoo.com.br (G. C. P. Sella), marioferraz@yahoo.com.br (M. B. J. Ferraz) \\ *Corresponding author
}

\section{To cite this article:}

Guilherme Constante Preis Sella, Bárbara das Neves Linhares, Mario Bazanelli Junqueira Ferraz. Modified Lateral Crural Tensioning: Improving the Tip Without Articulated Alar Rim Grafts. Journal of Surgery. Vol. 9, No. 5, 2021, pp. 220-225. doi: 10.11648/j.js.20210905.11

Received: August 13, 2021; Accepted: August 27, 2021; Published: September 4, 2021

\begin{abstract}
Objective: Lateral Crural Tensioning (LCT) is an important technique to refine the tip. However, a frequent problem that can occur is the weakening of the External Nasal Valve (ENV) and the constant use of Articulated Alar Rim Graft (AARG). The aim of this study is to show the results of the same surgeon (MBJZ) within one year of the Modified LCT, and review of the literature. Methods: We analyzes technical details of 149 patients that underwent rhinoplasty in one year (between July 1, 2018 and June 30, 2019) and had at least 1 year of follow-up. Results: Out of 144 patients, 25 (17.36\%) were revision surgery. In 114 cases $(79.17 \%)$ only the septum was used; and Septal Extension Graft (SEG) was performed in 142 of the 144 patients $(98.61 \%)$. Of these, $130(91.55 \%)$ were latero-lateral and $12(8.45 \%)$ end-to-end. And we found that the modified LCT was used in 136 (94.44\%) of the 144 patients; in these, 73 (53.68\%) it was necessary to use Floating Alar Rim Graft (FARG) and 5 (3.68\%) AARG. Conclusion: we demonstrate a technique that reduce the need for resection of inferior cartilages, the use of grafts and the improvement of nasal function, with strengthening of the ENV without the need to reconstruct the alar border using contour grafts.
\end{abstract}

Keywords: Rhinoplasty, Lateral Crural Tensioning, External Nasal Valve, Septal Extension Graft, Alar-contour Grafts

\section{Introduction}

Rhinoplasty remains one of the most difficult surgeries, because the nose must have a natural appearance, with good symmetry, projection, length and rotation. And a more refined nasal tip can be created on a slightly concave dorsum that continues with the tip without a visible transition, with the supratip break the more caudal possible. [1]

Another important characteristic of the tip is a smooth transition from tip lobule to the alae. The soft triangle usually undergoes important changes in the late postoperative period. Thus, several types of techniques and use of grafts have already been described; many of the deformities observed after rhinoplasty are due to incisions, sutures or exaggerated resections of the lower cartilages, leading to clamping, asymmetries, retractions of the nasal tip and the relationships between lobule, columella and alae.
Lateral Crural Tensioning (LCT), described by Davis [2], has gained prominence in contemporary rhinoplasty due to the consistent results; however, a frequent problem that can occur is the weakening of the soft triangle region and collapse of the External Nasal Valve (ENV). A 1-2mm retraction in this region can lead to a concavity of the alar contour, isolating the tip causing pinching of the region, leading to a globular aspect of the tip, and, possibly, nasal obstruction. [1, 3]

Therefore, it is very important that surgical techniques should achieve harmony and stability in this region, and a way to avoid this is through the use of alar-contour grafts, specially the Articulated Alar Rim Graft (AARG) [2, 4-9]; however, some disadvantages can occur with its use. Our objective is to present a description of a technique to refine and structure the nasal tip, which is a modification of the LCT, and we will analyze the surgical technique of patients that have done surgery within one year of the same surgeon. 
In addition, we will do an extensive literature review on the topic.

\section{Methods}

The surgical files of patients submitted to rhinoplasty by the same senior surgeon (MBJF) between July 1, 2018 and June 30, 2019 were verified. The results were analyzed and presented together with a review of the surgical techniques already described in the literature for the correction aestheticfunctional surgical procedure of the nasal tip. Such surgical techniques are used routinely by the authors of the article in patients in private practice and is intended for primary surgery but can also be used in selected cases of revision rhinoplasty. This study was performed according to the standards for biomedical research on human subjects set by the Declaration of Helsinki.

\section{Results}

Through the search of the surgical files, we found that 149 patients underwent rhinoplasty between July 1, 2018 and June 30, 2019; however, 5 records were not recovered due to system problems and, therefore, we will analyze the data of 144 patients. Of these, the average age was 29.85 years, with a minimum of 16 years and a maximum of 55 years; 130 patients were female $(93.06 \%)$ and $10(6.94 \%)$ male. We found that primary rhinoplasty corresponded to 119 (82.64\%) cases and revision surgery to $25(17.36 \%)$ - 2 of which were from the surgeon himself. Other results found will be demonstrated throughout the text.

\section{Discussion}

Open approach is performed, with a transcolumellar incision and with marginal incisions that extend to the most lateral curvature of the lateral crura. The soft tissue is conventionally elevated through a supraperichondrial plane out of the osteocartilaginous structure, exposing the lower, upper cartilages and the nasal dorsum. The nasal septum is dissected in a subperichondrial-subperiosteal plane along its entire length.

Dorsum surgery (enlargement or reduction) and bone fracture are performed. For dorsum reduction we use both techniques: resection and reconstruction (structured) of the dorsum or preservation (as S.P.A.R. [10] or "push/let down" [11]), and this does not interfere in tip surgery. We found that 118 of the 144 surgeries (81.94\%) used Structured rhinoplasty, 18 cases (12.5\%) S.P.A.R. and 8 cases (5.56\%) osteoplasty. As the objective here is the specific report of the nasal tip, we will not stick to the dorsum technique.

SEG can be defined as any graft that is attached to the septum and the lower cartilages. It was first described by Toriumi [12] in 1995, however Byrd et al. [13] was another author with an important contribution at the same time. It is usually harvested from the quadrangular cartilage of the nasal septum with or without the Perpendicular Plate of the
Ethmoid (PPE) or Vomer, which is ideal due to its adequate thickness, flat shape, strength and resistance. However, if septal cartilage is limited, the graft can be harvested from the cavum concha or rib cartilage. In this circumstance, the authors' preference is usually the rib cartilage with cuts in the oblique direction [14], especially in cases of revision, as the ear cartilage is curved and weak, not being a good cartilage for structuring. Regarding the use of grafts in surgery, we found that in 114 cases (79.17\%) only the septum was used (with or without PPE); in 18 (12.5\%) rib cartilage was necessary; 16 (11.11\%) ear cartilage and 3 (2.08\%) use of the temporal fascia.

The LCT requires a stable central point to connect the new domus that will be created later. This usually involves placing a SEG, but a straightforward Tongue-In-Groove (TIG) [15] approach can be performed occasionally in the case of a tension nose or pendant columella that needs stabilization of the tip or reduction of the projection. The position of the SEG will configure the stability of the nasal tip, tip projection, nose rotation, naso-labial angle, nostril shape, relation of the tip to the nasal dorsum, supratip break, infratip break point, columella thickness and the positioning between alarcolumella.

There are basically two ways of fixing the graft to the septum: end-to-end or side-to-side (also called overlapping). End-to-end fixation is not so firm, and it could move from the midline or may lose projection of the nasal tip, but it can be used in the case of little cartilage available [16] or very short septum.

There is evidence that the support force in the latero-lateral technique is more stable than the end-to-end technique [17, 18]; thus, latero-lateral fixation has been preferred by the authors because of its greater ease of fixation and greater stability, with more tension being applied if desired. However, care must be taken with the positioning of the graft and its thickness, as a risk of this technique is the narrowing of the Internal Nasal Valve (INV) that can occur in these cases.

SEG was performed in 142 of the 144 patients (98.61\%); only 2 cases (1.39\%) the TIG was performed. Of these, 130 (91.55\%) were latero-lateral and 12 (8.45\%) end-to-end. Of the latero-lateral cases, 110 (84.61\%) were positioned left of the septum, $19(14.62 \%)$ to the right and only 1 case (0.77\%) was bilateral.

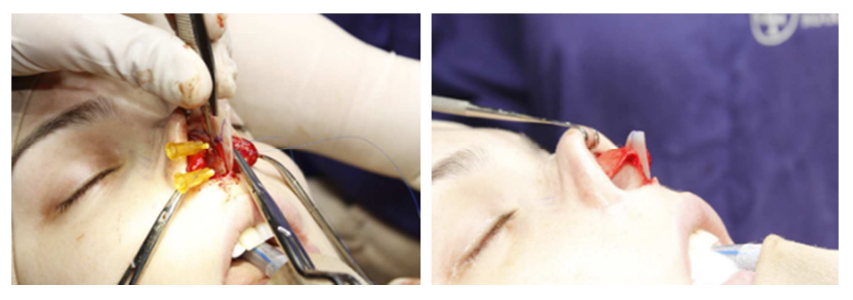

Figure 1. (A) SEG stabilized in the proper position; (B) SEG already sutured.

A slightly larger than necessary SEG can be initially sutured in the native septum and the antero-caudal region can subsequently be trimmed to the appropriate size as needed (Figure 1A, B). The ideal SEG should be slightly less projected tha $\mathrm{n}$ the new domus (about $1-2 \mathrm{~mm}$ ), in addition to 
being fixed on the medial crura and septum - it should involve the Anterior Septal Angle (ASA), further stabilizing the graft, which reduces the risk of loss of projection.

In cases that a firm and projected nasal tip is desired (as in Asian noses, afrodescendants and with retropositioning of the maxilla), SEG can be supported in the premaxilla. However, this is an ideal model for correcting major deformities; often the SEG is only fixed in the septum, in which case the greater the overlap with the septum (at least $4 \mathrm{~mm}$ ) [16] lower the risk of tip ptosis in the postoperative period. We only loosen and re-fix the septum of the Anterior Nasal Spine (ANS) in case of caudal deviation; there is no consensus in the literature on the type of thread used for this fixation (whether absorbable or non-absorbable). [3, 19]

For a long time, through different techniques, it has been shown that the tip bulbosity is due to the convexity of the lower cartilages and not their size. [1, 2, 20] There is sufficient evidence in the literature to demonstrate that resection of the cephalic portion of the lateral crura does not make the tip thinner, but only weakens it with consequent lobular clamping, alar retraction, asymmetry, excessive tip rotation, unwanted loss of tip projection and / or symptomatic collapse of the nasal valve. [1, 2, 9, 21-23]

Described by Kridel [20], Lateral Crura Steal (LCS) is a simple and effective surgical maneuver that results in the refinement of the nasal tip: relocating the domus. Most recently Davis [2] described the LCS associated with the SEG as the Lateral Crural Tensioning (LCT), an even more powerful way of refining the nasal tip. In this technique, an important lateralization of the domus associated with SEG fixation is performed; for this, a central and very fixed SEG is necessary, so that the tip does not over rotate - which is why it is not possible to perform this maneuver properly when using columellar strut. Thus, the cartilage redistribution of the tip is performed without removing large segments of the cephalic margin or vertically incising the lateral crura, but simply relocating the new domus, which delineates the point of definition of the tip. The goal is to make the lateral crura as tense as possible without deforming them; and the recruitment of lateral crural tissue shortens the lateral crural, creates tension and reduces its convexity.

As there is a domal steal, there is an increase in the length of the medial crura; and it can be freely dissected along its length and repositioned more inferiorly. In extreme cases, resection or overlapping of the medial crural can be performed. Care must be taken because if there is too much lateralization of the domus the medial crura may increase with a consequent increase in the lobular component of the tip. The medial crura, together with the recruited tissue, is then sutured to the SEG, and the "double break columellar point", columellar show and the subnasal point can be precisely constructed.

Among the functional and aesthetic benefits of LCT $[2,20$, $24]$ we can mention the reduction and refinement of the nasal tip and the increase of rotation and projection of the nasal tip. As the nose is a pyramid, in important increases in the projection of the tip (such as African, Asian and retro- positioned maxilla), there is a reduction in the width of the nasal base, and there is often no need for an incision to reduce this region.

There are several other ways to strengthen the lateral nasal wall and refine the tip [25]; the great advantage of Modified LCT over Lateral Crura Strut Graft [26] and to Batten Graft [27] it is not usually necessary to use grafts, since there is often no material available, in addition to the risk of asymmetry of the nasal tip. Another disadvantage of these grafts is the possibility of visualization (in thin-skinned patients) or obstruction of INV if misplaced.

Davis [2] describes the LCT associated with SEG, however it mentions that a problem that can occur in the postoperative period is the collapse of the ENV and alar retraction; other authors who have also been using this technique report the same problem [6, 7]. And they all mention that prevention / treatment must be carried out with the use of alar contour grafts [2, 4-9, 28, 29]. These types of grafts were first described by Troell et al. [30] who reported a $94.9 \%$ improvement in obstruction resulting from the collapse of the nasal valve, and after improvement by other authors. [31, 32] They can be Floating Alar Rim Graft (FARG), when it is only in an intra-dermal pocket; or Articulated (AARG) when they are fixed medially in the lateral crura and positioned in a pocket inside the skin laterally. It is a very useful graft, with multiple indications [33], easy to perform and requires little material; however, it can lead to bulbous tip and enlargement of the nasal lobe/alar complex, in addition to the risk of asymmetry, visibility in the skin and extrusion (if not well fixed).

Thus, an effective method to avoid the collapse of the ENV and to reinforce the transition from the tip lobe to the alar lobe is to perform the Modified LCT. This can be achieved through the tensioning of the lateral crura associated with their rotation around a horizontal axis, causing the caudal margin to be close to the same level as the cephalic margin, what is well known in the literature as the resting angle concept [34]. The closer these margins are, the more stable the ENV will be and the lesser the possibility of using graft with alarmed contours (specially the AARG), the transition from the earlobe to the alar lobe being more natural. And there are some sutures described in the literature to achieve this, all of which have the same goal.

One way is through oblique sutures of the new domus; this was a concept introduced in 2006 by Toriumi [1] and reinforced by the same recently [3]. It is performed so that the passage of the medial suture is made as close as possible to the caudal margin of the domus and the lateral passage is made as close to the cephalic edge of the cartilage.

Çakir et al. [34] described the cephalic domus suture, in which sutures are performed on the cephalic edges of the medial and lateral crura, 2 to $3 \mathrm{~mm}$ posterior to the dome. And another way to achieve this same effect is through the Cranial Tip Suture, described by Kovacevic and Wurm. [35] They report a suture that crosses the medial border of the intermediate crus to the cephalic margin of the lateral crura to rotate it in a more favorable orientation, and with the second 
"bite" flattening it, forming a "triangle".

When performing the Modified LCT using a SEG to suspend the lower cartilages, considerable tension can be generated at the suture fixation point. To perform this step, the surgeon comes to the patient's bedside and has a superior view of the cartilages, in order to facilitate the visualization of how much will be lateralized (Figure 2A). The recruitment amount is typically 3 to $5 \mathrm{~mm}$ but can be greater or less depending on the degree of curvature reduction required. It is extremely important to balance forces in the best possible way between the left and right sides so as not to cause asymmetries (Figure 2B). To ensure that the new tip is stable and properly aligned, it is essential that the septum-SEG complex is flat and rigid.

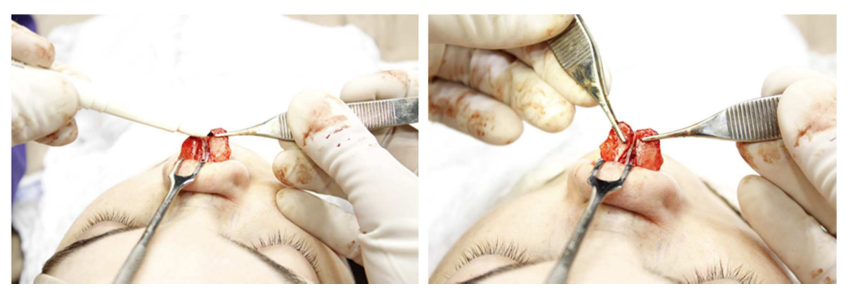

Figure 2. (A) tensioning the right lower cartilage; (B) tensioning and symmetrization of the lower cartilages for making the new domus.

Generally, what we do is fix the new domus in the desired location with the tensioning of the lateral crura, and then we perform an oblique suture, so that the caudal margin of the lateral crura will move anteriorly and the cephalic margin will move later (Figure $3 \mathrm{~A}$ and $3 \mathrm{~B}$ ).
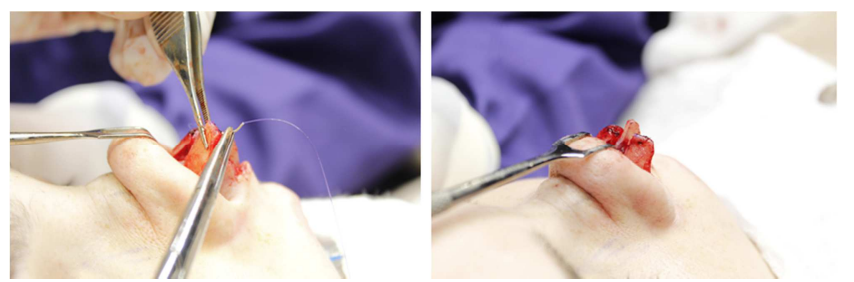

Figure 3. (A) Execution of the oblique suture in the domus; (B) and rotation of the lateral crura on the horizontal axis, with the caudal edge very close to the cephalic edge.

If the cartilages do not reach the proper position, cephalic stitches can be performed. And a small cephalic portion of the domus can still be resected at this stage (paradomal resection or "dog ear"). The new domes (on both sides) are attached to the SEG or septum (TIG) in a single suture, so that the point of definition of the tip is at the location of the most caudal point of the new domes (Figure 4).

As a result of the Modified LCT, the cephalic border is at the same height as the cranial border in the lateral crura, and there is no need to do a cephalic trim. Thus, the important point in this maneuver is not the removal of cartilage, but its new orientation. Another advantage of this technique is that the supratip break will be closer to the tip, making it more refined. ${ }^{1}$ As a result, we will have vectors of strength from different directions in the tip (Figure 5).
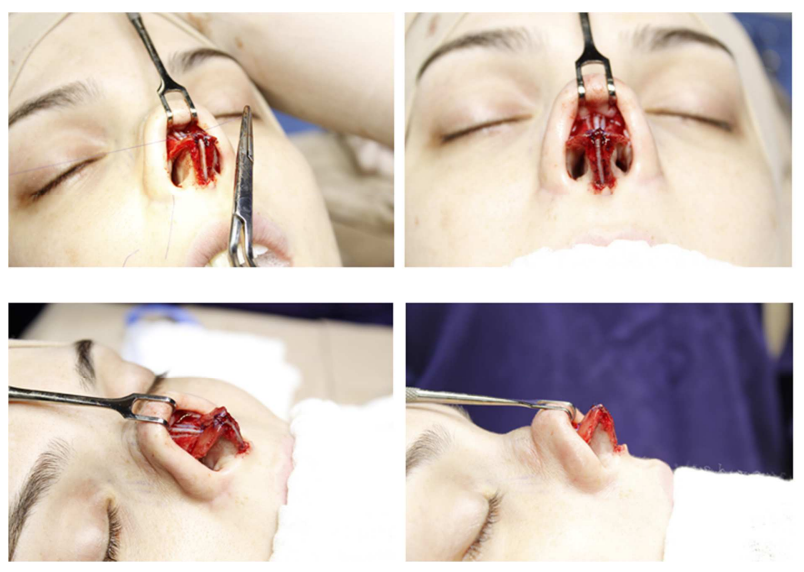

Figure 4. A)/B)/C)/D) Final aspect of the Modified LCT.
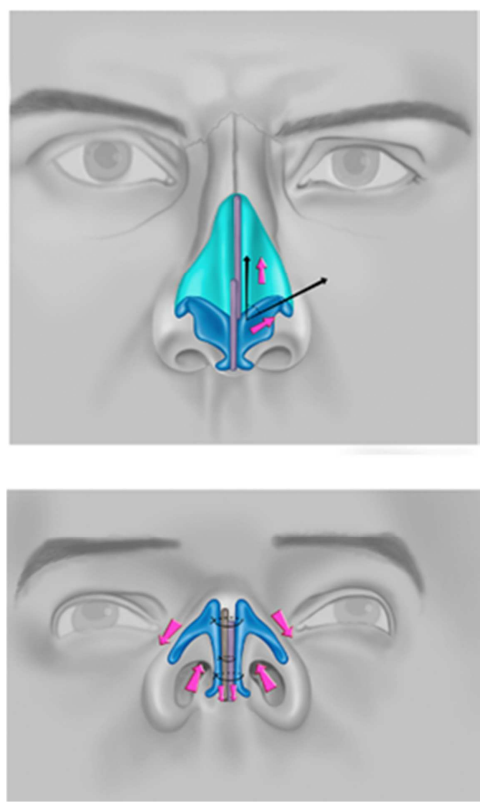

Figure 5. A)/B) Vectors of tensioning in LCT.

Kosins and Daniel [36] reported that they reduced the use of graft with alar contours from $80 \%$ to $16 \%$ with LCT associated with tip cranial suture and/or cephalic domus sutures. And when these are needed, they are for an aesthetic option or to correct small asymmetries and positioned as FARG. And we found that the modified LCT was used in 136 (94.44\%) of the 144 patients; in these, 73 (53.68\%) it was necessary to use FARG and 5 (3.68\%) AARG. Of the 8 (5.56\%) patients who did not use the LCT, 3 were using FARG and only one AARG. Overall, 76 of the 144 (52.78\%) required bilateral contour alar and 10 (6.94\%) unilateral alar contour.

\section{Conclusion}

What the authors are demonstrating here is a combination of simple techniques that, if used properly, achieve a stable and predictable result. The use of the SEG sutured laterally to the septum provides a firm and stable central septum-SEG set, with the structure of the new tip on which the tensioning technique can be performed. And then the sutures in the new 
domus lead to the modified LCT, causing the cranial edges to be on the same level as the caudal edges, strengthening the region of the ENV and the transition from the tip lobe to the alar lobe.

The advantages of this technique include the need for little resection of inferior cartilages, the use of few grafts and the improvement of nasal function, with strengthening of the ENV without the need to reconstruct the alar border using contour grafts.

\section{Conflict of Interest}

All the authors do not have any possible conflicts of interest.

\section{References}

[1] Toriumi DM. New concepts in nasal tip contouring. Arch Facial Plast Surg 2006; 8 (3): 156-185.

[2] Davis RE. Lateral crural tensioning for refinement of the wide and underprojected nasal tip: rethinking the lateral crural steal. Facial Plast Surg Clin North Am 2015; 23 (1): 23-53.

[3] Toriumi DM. Nasal tip contouring: anatomic basis for management. Facial Plast Surg Aesthet Med 2020; 22 (1): 1024 .

[4] Davis RE. Nasal tip complications. Facial Plast Surg. 2012; 28 (3): 294-302.

[5] Davis RE. Revision of the overresected nasal tip complex. Facial Plast Surg 2012; 28 (4): 427-439.

[6] Foulad A, Volgger V, Wong B. Lateral crural tensioning for refinement of the nasal tip and increasing alar stability: a case series. Facial Plast Surg 2017; 33 (3): 316-323.

[7] Calloway HE, Heilbronn CM, Gu JT, Pham TT, Barnes CH, Wong BJF. Functional outcomes, quantitative morphometry, and aesthetic analysis of articulated alar rim grafts in septorhinoplasty. JAMA Facial Plast Surg 2019; 21 (6): 558565 .

[8] Kao WTK, Davis RE. Postsurgical alar retraction: etiology and treatment. Facial Plast Surg Clin North Am 2019; 27 (4): 491-504.

[9] Davis RE, Ostby ET. How to create ideal alar form and function. Facial Plast Surg 2020; 36 (1): 034-045.

[10] Ferraz MBJ, Zappelini CEM, Carvalho GM De, Guimaraes AC, Chone CT, Dewes W. Conservative surgery of the nasal dorsum - the philosophy of the septal pyramid adjusting and repositioning (S.P.A).). Rev Bras Cir Cabeça Pescoço 2013; 42 (3): 124-130.

[11] Saban Y, Daniel RK, Polselli R, Trapasso M, Palhazi P. Dorsal preservation: The push down technique reassessed. Aesthetic Surg J 2018; 38 (2): 117-131.

[12] Toriumi DM. Caudal septal extension graft for correction of the retracted columella. Oper Tech Otolaryngol - Head Neck Surg 1995; 6 (4): 311-318.

[13] Byrd HS, Andochick S, Copit S, Walton KG. Septal extension grafts: a method of controlling tip projection shape. Plast Reconstr Surg 1997; 100 (4): 999-1010.

[14] Tastan E, Yucel OT, Aydin E, Aydogan F, Beriat K, Ulusoy MG. The oblique split method: A novel technique for carving costal cartilage grafts. JAMA Facial Plast Surg 2013; 15 (3): 198-203.

[15] Kridel RW, Scott BA, Foda HM. The tongue-in-groove technique in septorhinoplasty. A 10-year experience. Arch Facial Plast Surg 1999; 1 (4): 246-256; discussion 257-258.

[16] Kim MH, Choi JH, Kim MS, Kim SK, Lee KC. An introduction to the septal extension graft. Arch Plast Surg 2014; 41 (1): 29-34.

[17] Hwang SH, Hwang K. Supporting strength of septal extension grafts. J Craniofac Surg 2011; 22 (6): 2323-2326.

[18] Jeong JY. Obtaining maximal stability with a septal extension technique in East Asian rhinoplasty. Arch Plast Surg 2014; 41 (1): $19-28$.

[19] Benavides G, Villate P, Malaver C. Caudal septal extension graft sutured with absorbable material and not fixed to the nasal spine region compared with the conventional fixation method: A retrospective study. Aesthetic Plast Surg 2019; 43 (3): 759-767.

[20] Kridel R W, Konior RJ, Shumrick KA, Wright WK. Advances in nasal tip surgery. The lateral crural steal Arch Otolaryngol Head Neck Surg 1989; 115 (10): 1206-1212.

[21] Gubisch W, Eichhorn-Sens J. Overresection of the lower lateral cartilages: A common conceptual mistake with functional and aesthetic consequences. Aesthetic Plast Surg 2009; 33 (1): 6-13.

[22] Alexander AJ, Shah AR, Constantinides MS. Alar retraction: Etiology, treatment, and prevention. JAMA Facial Plast Surg 2013; 15 (4): 268-274.

[23] Nishoka GJ, Cook PR, Davis WE, McKinsey JP. Functional endoscopic sinus surgery in patients with chronic sinusitis and asthma. Otolaryngol Neck Surg 1994; 110 (6): 494-500.

[24] Foda HMT. Cosmetic management of the droopy tip: A comparison of three alar cartilage - modifying techniques. Plast Reconstr Surg 2003; 112 (5): 1408-1417; discussion 1418-1421.

[25] Vaezeafshar R, Moubayed SP, Most SP. Repair of lateral wall insufficiency. JAMA Facial Plast Surg 2018; 20 (2): 111-115.

[26] Gunter JP, Friedman RM. Lateral crural strut graft: Technique and clinical applications in rhinoplasty. Plast Reconstr Surg 1997; 99 (4): 943-955.

[27] Park SS, Hughley BB. Revision of the functionally devastated nasal airway. Facial Plast Surg 2012; 28 (4): 398-406.

[28] Ballin AC, Kim H, Chance E, Davis RE. The articulated alar rim graft: reengineering the conventional alar rim graft for improved contour and support. Facial Plast Surg 2016; 32 (4): 384-397.

[29] Goodrich JL, Wong BJF. Optimizing the soft tissue triangle, alar margin furrow, and alar ridge aesthetics: analysis and use of the articulate alar rim graft. Facial Plast Surg 2016; 32 (6): 646-655. 
[30] Troell RJ, Powell NB, Riley RW, Li KK. Evaluation of a new procedure for nasal alar rim and valve collapse: Nasal alar rim reconstruction. Otolaryngol - Head Neck Surg 2000; 122 (2): 204-211.

[31] Rohrich RJ, Raniere J, Ha RY. The alar contour graft: Correction and prevention of alar rim deformities in rhinoplasty. Plast Reconstr Surg 2002; 109 (7): 2495-2505.

[32] Guyuron B. Alar rim deformities. Plast Reconstr Surg. 2001; 107 (3): 856-863.

[33] Boahene KDO, Hilger PA. Alar rim grafting in rhinoplasty:
Indications, technique, and outcomes. Arch Facial Plast Surg 2009; 11 (5): 285-289.

[34] Çakir B, Do an T, Örero lu AR, Daniel RK. Rhinoplasty: Surface aesthetics and surgical techniques. Aesthetic Surg J 2013; 33 (3): 363-375.

[35] Kovacevic M, Wurm J. Cranial tip suture in nasal tip contouring. Facial Plast Surg 2014; 30 (6): 681-687.

[36] Kosins AM, Daniel RK. Decision making in preservation rhinoplasty: A 100 case series with one-year follow-up. Aesthetic Surg J 2020; 40 (1): 34-48. 